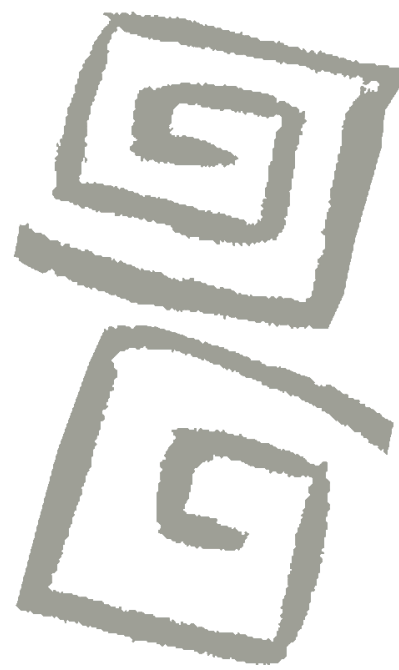

\title{
La salud de trabajadores inmigrantes haitianos en Mato Grosso, Brasil: vulnerabilidades y riesgos
}

\author{
The health of Haitian immigrant workers in Mato \\ Grosso, Brazil: vulnerabilities and risks
}

Luís Henrique da Costa Leão ${ }^{1}$, Ana Paula Muraro², Fabiano Tonaco Borges ${ }^{3}$, Jorge Mesquita Huet Machado ${ }^{4}$

'Psicólogo, doctor en Saúde Pública. Profesor Adjunto III, Instituto de Saúde Coletiva, Universidade Federal de Mato Grosso. Cuiabá, Mato Grosso, Brasil. $\square$ iD

${ }^{2}$ Nutricionista, doctora en Fisiopatología Clínica y Experimental. Profesora, Instituto de Saúde Coletiva Universidade Federal de Mato Grosso. Cuiabá, Mato Grosso, Brasil. $\bowtie$ iD

${ }^{3}$ Odontólogo, doctor en Odontología Preventiva y Social. Profesor, Instituto de Saúde Coletiva, Universidade Federal Fluminense, Brasil. $\triangle$ (iD)

${ }^{4}$ Médico, doctor en Saúde Pública. Coordinador Programa de Promoção da Saúde, Ambiente e Trabalho, Fundação Oswaldo Cruz. Brasília, Brasil. $\square$ (iD)
RESUMEN Se describen las relaciones entre inmigración, salud, trabajo y ambiente con el objetivo de analizar la inserción de inmigrantes haitianos en procesos productivos de Mato Grosso, destacando los riesgos para la salud y las vulnerabilidades socioambientales. Se trata de una investigación-acción desarrollada junto a la comunidad haitiana, organizaciones de la sociedad e instituciones del Estado. En 2014 y 2015 se aplicó un cuestionario a población haitiana de la ciudad de Cuiabá (capital del estado de Mato Grosso), para caracterizar sus condiciones de vida y trabajo. Además, se relevaron datos de distintas instituciones y el registro de empleadores que sometieron a trabajadores a condiciones de esclavitud contemporánea. Los resultados muestran 2.151 trabajadores haitianos registrados en el mercado formal de trabajo en 2014, distribuidos en 27 municipios de Mato Grosso. Dos sectores económicos se destacaron en la absorción de trabajadores haitianos: las industrias de transformación (principalmente frigoríficos) y la construcción civil. Entre los 452 haitianos entrevistados, el 52,7\% estaba trabajando y el 26,5\% mencionó una carga horaria semanal superior a 48 horas. El estudio indica la fragilidad de la inserción social de esta población, la cual se expresa en la presencia de haitianos en áreas y procesos productivos de alto riesgo socioambiental.

PALABRAS CLAVES Migrantes; Salud Laboral; Ambiente; Brasil.

\begin{abstract}
The relationships among immigration, health, work and environment are explored with the aim of analyzing the insertion of Haitian immigrants in the productive processes of Mato Grosso, highlighting health risks and socioenvironmental vulnerabilities. An action research study was carried out in conjunction with the Haitian community, social organizations and State institutions. In 2014 and 2015, a questionnaire was applied among the Haitian population of the city of Cuiabá (capital state of Mato Grosso) to characterize living and working conditions. In addition, data from different institutions and the registry of employers that submitted workers to conditions of modern-day slavery were analyzed. The results include 2,151 Haitian workers involved in the formal labor market in 2014, distributed in 27 municipalities of Mato Grosso. Two economic sectors in particular absorbed Haitian workers: transformative industries (especially meat processing plants) and civil construction. Among the 452 Haitians interviewed, 52.7\% were working and $26.5 \%$ reported a workweek longer than 48 hours. The study shows the fragility of the social insertion of this population, expressed through the presence of Haitians in areas and productive processes of high social risk.
\end{abstract}

KEY WORDS Migrants; Occupational Health; Environment; Brazil. 


\section{INTRODUCCIÓN}

La globalización económica y la nueva división internacional del trabajo genera intensos flujos financieros, materiales y poblacionales entre regiones y Estados nación que repercuten en forma directa en las características y perfiles del uso de la fuerza de trabajo. En Brasil, el número de inmigrantes en los procesos productivos ha crecido con la presencia de bolivianos, haitianos, peruanos, portugueses y españoles, convocados para trabajos específicos y calificados ${ }^{(1)}$ o por la libre demanda, ocupando diferentes funciones en los más variados sectores económicos.

La diáspora haitiana hacia la nación brasileña avanzó con mayor intensidad a partir de 2011 y tal movimiento migratorio ya superó las 39.000 personas $^{(2)}$ que tienen como destino, en su mayoría, estados del sur y del sudeste ${ }^{(3)}$.

Esta migración está ligada al dramático desastre ambiental de 2010, al apoyo humanitario ofrecido por Brasil a Haití y a las dinámicas del propio capitalismo global que ejercen una violencia estructural sobre poblaciones más vulnerables, por lo que es difícil que sobrevivan en la tierra natal( ${ }^{(4)}$, especialmente, en países periféricos.

A partir de 2012, el estado de Mato Grosso pasó a ser el centro de las inmigraciones haitianas, debido a su economía en expansión y al megaevento de la Copa del Mundo de 2014, que aumentó los flujos financieros y generó altas demandas de trabajadores. A pesar de que diversas instituciones y movimientos sociales estaban involucrados en la acogida y apoyo a los haitianos, no hay una estimación del número de los inmigrantes haitianos en el estado, y los responsables de ejecutar la Política Nacional de Salud del Trabajador y la Trabajadora, no cuentan con información sobre la inserción de este nuevo grupo de trabajadores en los sectores productivos y en las regiones de su mayor incidencia.

Se destaca que, debido a los propios ciclos de reproducción del sistema capitalista, generalmente, los inmigrantes se instalan en las "economías grises de las grandes

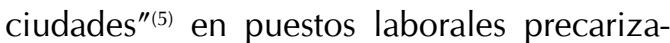
dos, plagados de riesgos, con salarios más bajos y menor reconocimiento social. Esta fuerza de trabajo representaría así al "trabajador perfecto" para las relaciones de trabajo capitalistas, justamente por ser "mercantilizado y explotable, flexible y descartable"(4). Históricamente, los inmigrantes configuraron una población desigualmente expuesta a riesgos ocupacionales y ambientales, a malas condiciones de trabajo, sujeta a violencia física y simbólica, y a recortes económicos y privación de derechos ${ }^{(6)}$. Las vulnerabilidades se expresan tanto en la esfera de la producción (condiciones y relaciones de trabajo) como en la esfera de la reproducción (alimentación, vivienda, acceso a la información y ocio) $)^{(7,8)}$

Ciertos estudios señalan cómo los trabajadores inmigrantes son expuestos a los agrotóxicos, a la sobreexplotación y a las jornadas exhaustivas, que caracterizan las formas de trabajo esclavo contemporáneo ${ }^{(9,10,11)}$. La condición de inmigrante, sobre todo, la de los no documentados, genera una mayor vulnerabilidad a esas situaciones debido a los miedos que los silencian frente a los riesgos a los que están expuestos. No es extraña la omisión de accidentes y agravios derivados del trabajo ${ }^{(6,7,8)}$. Por estas razones, los inmigrantes tienen mayores posibilidades de morir en el trabajo ${ }^{(12)}$. Sin embargo, los impactos directos de la inmigración en la salud aún son poco conocidos, como la relación de las condiciones de trabajo con las inequidades en salud ${ }^{(7)}$.

Al considerar la centralidad de la categoría trabajo tanto en los flujos migratorios como en el proceso salud-enfermedad, y seguir los marcos teóricos del campo de la salud del trabajador, cabe preguntarse cuán determinante y condicionante del proceso salud-enfermedad es la inserción de este nuevo grupo de trabajadores al proceso de trabajo ${ }^{(13,14)}$.

Resulta necesario, por lo tanto, conocer mejor las condiciones de vida y trabajo, sobre todo, cuando comienzan a surgir casos de violencias, actitudes xenofóbicas, accidentes de trabajo y esclavitud contemporánea en esa población, en Brasil y en Mato Grosso. 
El objetivo de este artículo fue describir la inserción de inmigrantes haitianos en procesos productivos de Mato Grosso, destacando los riesgos para la salud y las vulnerabilidades socioambientales.

Este trabajo es parte de una investigación mayor titulada "Estudo da migração haitiana para Mato Grosso: etno-história, perfil socioeconômico, condições de saúde e acesso ao SUS", de la cual fueron publicados los artículos "Migração internacional, saúde e trabalho: uma análisis sobre os haitianos em Mato Grosso, Brasil"(15), Socioeconomic and health profile of Haitian immigrants in a Brazilian Amazon State ${ }^{(16)}$ y Prenatal followup of Haitian and Brazilian women in Mato Grosso $^{(17)}$ en los que se abordan otras dimensiones de análisis.

\section{RECORRIDO TEÓRICO- METODOLÓGICO}

Para discutir elementos de la dinámica de inserción de los inmigrantes haitianos en procesos productivos del territorio matogrossense se adoptó, como perspectiva crítica de análisis, la lógica de predecir impactos en la salud frente a la dinámica económicoproductiva de Mato Grosso, sin la pretensión de cuantificar la exposición a riesgos ocupacionales y a situaciones de vulnerabilidad socioambiental. Los riesgos y las vulnerabilidades en el ámbito de las relaciones saludtrabajo-ambiente no se utilizan aquí con un sentido reduccionista, tecnicista o biomédico, sino en sus dimensiones ampliadas ${ }^{(18)}$. En ese sentido, se valoran procesos históricos que moldean los condicionantes de los riesgos generados por los modelos de desarrollo económico y se busca visualizar la distribución espacial de grupos poblacionales pobres y con privaciones, que viven o circulan en áreas de riesgo o de degradación ambien$\mathrm{tal}^{(19)}$. Este punto de vista comprende que los problemas socioambientales derivan "del actual modelo de desarrollo económico, de los procesos de deslocalización y desregulación, que intensifican las relaciones entre grupos vulnerables y áreas de riesgo ambiental"(19). O sea, en comunidades, en determinados grupos $\mathrm{O}$, incluso, en ciudades enteras se asientan emprendimientos económicos extremamente peligrosos, porque el desarrollo económico genera en su seno ciclos perversos de exposición ambiental y ocupacional, además de violaciones a los derechos humanos, a la salud y al ambiente saludable ${ }^{(18)}$. En ese contexto, se alteran territorios que pasan a amparar sistemas socio-técnicos ambientales peligrosos que quedan sujetos a los ciclos de generación, exposición y efectos relacionados con los riesgos ambientales y ocupacionales $^{(18)}$. Las cargas y efectos nocivos de ese proceso, entretanto, se distribuyen de forma desigual, y recaen de modo más agudo sobre grupos más pobres e históricamente excluidos.

En este artículo nos limitamos a identificar sistemas sociotécnicos ambientales peligrosos, considerando el alto índice de uso de agrotóxicos y los contextos de exposición a la situación de sometimiento de los trabajadores a condiciones análogas a la esclavitud. Reconocer la presencia de inmigrantes haitianos en procesos productivos de alto riesgo, en territorios actualmente expuestos a químicos extremadamente perjudiciales para la salud y el medioambiente, en lugares de histórica explotación de mano de obra, análoga a las formas de producción esclavista, posibilita observar las interrelaciones entre sistemas productivos y daños a la salud. $\mathrm{Si}$ bien la identificación de eventos particulares de cada exposición ha sido inviable en esta investigación, no buscamos sustentar una relación de causalidad lineal/objetiva, sino señalar situaciones de riesgo generadas por la exposición a condicionantes ocupacionales y socioambientales que, a su vez, impactan en la salud y debilitan la fuerza de trabajo.

Utilizamos la noción de vulnerabilidad de grupos humanos desde el punto de vista de las ciencias sociales y humanas en salud, que valoriza el proceso histórico de producción de grupos vulnerabilizados. Esa noción abarca la discriminación por clase, género, etnia, color y las relaciones de trabajo autoritarias, la precarización, los límites en la 
percepción de los riesgos y la capacidad de organización y movilización para el enfrentamiento del contexto de riesgo ${ }^{(18)}$. Al mismo tiempo, buscamos vislumbrar la expresión de las desigualdades sobre el grupo de haitianos inmigrantes, identificando la discriminación y la violencia simbólica en la inserción de ese grupo en determinados sectores y actividades.

La violencia simbólica se manifiesta, muchas veces, de modo velado en las relaciones sociales y se fundamenta en una dominación frecuentemente naturalizada que legitima a un determinado grupo poblacional, una práctica o un discurso. Deviene de imposiciones legitimadas que refuerzan "el poder arbitrario que la establece y que ella disimula"(20). La discriminación en sus múltiples formas (interpersonal, institucional, estructural, entre otras) se expresa también en las violencias perpetradas por grupos dominantes, basadas en diferencias de género, color o clase, que fundamentan ideologías de superioridad/ inferioridad $^{(21)}$ que, muchas veces, generan modalidades de explotación diferenciadas con impactos en la salud física y mental. Así, en ese artículo, se optó también por indagar las manifestaciones de sufrimiento (como dolores, angustias, miedos, sentimientos de desvalorización y de no reconocimiento), a partir de nociones de la psicodinámica dejouriana.

Según esta perspectiva, el trabajo nunca es neutro con relación a la salud, es una actividad humana compleja e intersubjetiva, movilizadora de dinámicas colectivas como vivenciar el sufrimiento, crear estrategias defensivas y reglas de trabajo que se originan al confrontar la práctica de los trabajadores con el trabajo real ${ }^{(22)}$. Por eso, es un importante mediador de la salud mental, que puede generar no solo sufrimientos psíquicos y enfermedades mentales, sino también placer y bienestar, en la medida que es central para la identidad y realización de los trabajadores ${ }^{(22)}$.

A partir de estas perspectivas teóricas se realizó una investigación-acción desarrollada en la capital matogrossense que buscó investigar diversos aspectos de las condiciones de vida y trabajo de haitianos para mediar el desarrollo de políticas públicas de promoción de la salud y ciudadanía. El proceso fue
Ilevado a cabo por un equipo interdisciplinario, en sucesivas aproximaciones al grupo de inmigrantes, inicialmente alojados en el Centro Pastoral para Migrantes (CPM) de Cuiabá y en la "Vila dos haitianos", microrregión de la ciudad donde reside una significativa proporción de haitianos.

La investigación-acción recolectó múltiples informaciones provenientes de varias fuentes de datos, como documentos públicos, grupos de conversaciones temáticas, entrevistas individuales abiertas, y también de un cuestionario desarrollado por el equipo de investigación con base en contactos de informantes claves, que fue aplicado a la población haitiana en Cuiabá y Várzea Grande, entre diciembre de 2014 y febrero de 2015. Contenía preguntas cerradas y abiertas dirigidas a caracterizar la población de inmigrantes haitianos en Cuiabá y Várzea Grande, con datos demográficos, socioeconómicos, y sobre el flujo migratorio, relativas a la actividad ejercida en Haití antes de migrar y al trabajo que ejercían en el momento de la entrevista, incluyendo información sobre exposición a riesgos ocupacionales, caracterización de las condiciones/relaciones de trabajo y manifestaciones de sufrimiento. Las preguntas abiertas permitieron obtener una visión de los haitianos sobre los procesos en los que están insertos, a partir de sus propios saberes y experiencias.

El cuestionario fue aplicado en una muestra probabilística y proporcional por sexo. Para el cálculo de la muestra se adoptaron los procedimientos de Lwanga y Lemeshow $^{(23)}$, y se partió del registro de entrada de inmigrantes haitianos en Cuiabá a cargo del Centro Pastoral para Migrantes, realizado entre 2012 y 2014 . Considerando que el número de haitianos registrados fue de 1.059 (16\% mujeres, prevalencia del $50 \%$, nivel de significancia del $95 \%$ y un error tolerable del $4 \%$ ), se llegó a 383 individuos. Para compensar posibles pérdidas, el tamaño muestral se acrecentó un 15\% (57 individuos). La población de estudio quedó conformada por 440 individuos y, según la proporcionalidad por sexo, la muestra debería estar compuesta por 370 hombres y 70 mujeres $^{(15)}$. La recolección 
de los datos se realizó entre diciembre de 2014 y febrero de 2017. Las entrevistas estuvieron a cargo de personas bilingües (criollo haitiano-portugués), debidamente capacitadas y bajo la supervisión de los investigadores responsables $^{(15)}$. Los sujetos de investigación fueron contactados por teléfono o personalmente e invitados a participar de la investigación (los contactos telefónicos y direcciones se obtuvieron en el Centro Pastoral para Migrantes ${ }^{(15)}$.

A través de líderes religiosos y de la Organización de Soporte a las Actividades de los Haitianos en Brasil (OSAHB) se articularon encuentros y conversaciones grupales temáticas, con una participación promedio de 40 haitianos en cada encuentro. Los diálogos bilingües (criollo haitiano-portugués) versaron sobre temas relativos a la salud de la mujer, acceso al Sistema Único de Salud (SUS), inmunización en Brasil, salud del hombre, relaciones laborales y salud del trabajador. Esos encuentros formaron ricos espacios de intercambio de experiencias, diálogo, discusión de saberes y, sobre todo, para organización de demandas y reivindicaciones de los haitianos. Este proceso involucró, además del equipo de investigación, a agentes de la Secretaría Municipal de Salud, el Ministerio de Trabajo y Empleo, el Ministerio Público de Trabajo, la Pastoral del Migrante y la Comisión Pastoral de la Tierra. En muchos encuentros emergieron denuncias de negligencias patronales, exposición a riesgos ocupacionales y ambientales, privación de derechos laborales y previsionales, situaciones de asedio y violencia simbólica en el trabajo, accidentes y enfermedades ocupacionales. Tales situaciones pusieron en evidencia la necesidad de que el equipo de investigación analizara, de modo más profundo, el proceso de inserción de esa población en los procesos de trabajo, sobre todo considerando que muchos inmigrantes se trasladan rápidamente hacia el interior del estado de Mato Grosso.

A partir de entonces, el equipo buscó acceder a datos secundarios sobre situaciones de explotación extrema, tipificadas como trabajo esclavo contemporáneo, al interior del estado y también información sobre inserción de haitianos en territorios de alta exposición ambiental a agrotóxicos. Esos datos secundarios se obtuvieron de la Comisión Pastoral de la Tierra; del Informe Anual de Relaciones Sociales del Ministerio de Trabajo y Empleo de 2014; de registros del Centro Pastoral para Migrantes de Cuiabá, obtenidos entre noviembre de 2012 y diciembre de 2014; del Instituto de Defensa Agropecuaria (INDEA). Para delimitar el número de haitianos dentro del mercado formal de trabajo de Mato Grosso, según municipio y sector económico, se analizaron datos de la Relación Anual de Informaciones Sociales (RAIS), disponibles en la página web del Ministerio de Trabajo ${ }^{(24)}$, la Clasificación Nacional de Actividades Económicas (CNAE) establecida por la Resolución CONCLA N ${ }^{\circ} 2$ del 25 de junio de $2010^{(25)} \mathrm{y}$ la Clasificación Brasileña de Ocupaciones de $2002^{(26)}$. Debido a las diferencias respecto de los sectores generadores de ingresos en los municipios, se consideró relevante caracterizar los principales sectores económicos en los que se insertaban los trabajadores haitianos, según capital y municipios del interior del estado. En este análisis, se consideró como capital los municipios de Cuiabá y Várzea Grande, que conforman la Región Metropolitana del Vale del Rio Cuiabá.

Considerando las disparidades regionales del estado y el hecho de que Mato Grosso es uno de los estados brasileños en los que predomina el agronegocio y el uso intenso de agrotóxicos en procesos productivos agrícolas, como también el alto índice de ocurrencias de trabajo esclavo contemporáneo, se identificaron municipios que avalaban el uso de agrotóxicos y en los que se produjeron denuncias y/o casos confirmados de trabajo esclavo. Los datos de las denuncias se obtuvieron a través de la Comisión Pastoral de la Tierra ${ }^{(27)} y$, los casos de rescate de trabajadores esclavizados, del registro de empleadores que sometieron trabajadores a la condición de esclavitud en el año 2015, divulgado por la División de Fiscalización para la Erradicación del Trabajo Esclavo (DETRAE) del Ministerio de Trabajo ${ }^{(28)}$.

En cuanto al uso de agrotóxicos en los municipios del estado, se analizó el banco 
de datos del Sistema de Información de Agrotóxicos (SIA) del Instituto de Defensa Agropecuaria que registra, desde 2005, los datos de los pedidos en las facturas emitidas por las casas revendedoras ${ }^{(29)}$. Este análisis se basó en la estrategia utilizada por Pignati et al..$^{(30)}$ que relacionaron los 10 municipios matogrossenses que más consumían agrotóxicos en 2012 con el proceso productivo agropecuario. Se consideró aquí un punto de corte de cuatro millones de litros para definir los municipios con mayor consumo de agrotóxicos.

El Centro Pastoral para Migrantes de la Archidiócesis de Cuiabá -principal responsable de la recepción de los inmigrantes en Mato Grosso- autorizó el acceso a los datos de los registros administrativos de todos los haitianos que fueron recibidos por la pastoral hasta diciembre de 2014. Estos datos se utilizaron para describir el flujo de inmigrantes en esa institución.

En cuanto a la tabulación y análisis de los datos, los datos recolectados a través del cuestionario y de los registros del Centro Pastoral para Migrantes pasaron por una doble digitación y validación en el programa Epi Info 7 y el programa SPSS (versión 17) para el análisis estadístico. Se utilizó el test de chicuadrado para probar las diferencias entre los grupos, y se adoptó una significancia del $5 \%$. La distribución espacial de los datos de la RAIS, los registros de los empleadores que sometieron trabajadores a esclavitud y del consumo de agrotóxico por municipio, se realizó con el auxilio del programa Tabwin del DATASUS.

La investigación fue aprobada por el Comité de Ética en Investigación con Seres Humanos del Hospital Universitário Julio Müller (No 29120414.0.0000.5541) y todos los participantes firmaron el consentimiento libre e informado antes de la recolección de datos. Este estudio fue financiado por la Fundação de Amparo a la Pesquisa do estado de Mato Grosso (FAPEMAT) (Proceso 155709/2014), y por el Conselho Nacional de Desenvolvimento Científico e Tecnológico (CNPq) (Proceso 445842/2014-7).

\section{RESULTADOS Y DISCUSIÓN}

\section{Flujo migratorio y situación socioeconómica de haitianos en Mato Grosso}

En Mato Grosso, los primeros haitianos Comenzaron a llegar en noviembre de 2012, y fueron recibidos por el Centro Pastoral para Migrantes de Cuiabá. Entre noviembre y diciembre de 2012, fueron recibidos solo seis inmigrantes haitianos; en 2013, 833 y en 2014 ese número descendió a 358, llegando a un total de 1.197 (84,3\% de sexo masculino). Entre mayo y agosto de 2013, se produjo el mayor flujo con el registro de 464 haitianos. Estas cantidades no reflejaban el número de nuevos inmigrantes en el estado, pues muchos se alojaban directamente en la casa de parientes y/o amigos ya residentes en la ciudad.

Los datos secundarios obtenidos indican un número mucho mayor. Al analizar la información de la RAIS, se verificó que hubo 2.151 registros de trabajadores haitianos en el mercado formal del estado de Mato Grosso en 2014, distribuidos en 27 municipios; siete de los cuales concentraron el 94,4\% de esos trabajadores: Cuiabá (1.264; $58,8 \%)$, Várzea Grande (277; $12,9 \%)$, Sorriso $(145 ; 6,7 \%)$, Sinop $(144 ; 6,7 \%)$, Rondonópolis (116; 5,4\%), Paranaíta (56; 2,6\%) y Nueva Mutum (28; $1,3 \%$ ). Por seis de esos municipios, pasa una de las principales vías de distribución de la producción del estado: la Ruta Federal BR 163.

Se entrevistó a 452 haitianos residentes en Cuiabá y Várzea Grande (373 hombres y 79 mujeres) y más de la mitad tenía menos de 35 años de edad. Aproximadamente 2/3 de los entrevistados estaban casados o en unión estable. Entre aquellos que mencionaron estar casados, el $60,7 \%$ de los hombres afirmaron que la compañera estaba en Haití y el $60,8 \%$ de las mujeres que vivían con sus parejas ${ }^{(15)}$. Este perfil demográfico se asemeja al verificado en otros estados brasileños ${ }^{(31,32)}$.

La mayoría de los entrevistados tenía ingresos superiores a un salario mínimo (49,3\%), 
con una mayor proporción de hombres $(p=<0,01)$, mientras el 30,1\% mencionó no tener ningún ingreso. Esto puede estar relacionado al hecho de que el 10,9\% $(\mathrm{n}=49)$ de los entrevistados habían Ilegado a Brasil hacía menos de 30 días cuando fueron entrevistados. O sea, vivían en el Centro Pastoral para Migrantes y todavía estaban sin empleo. Una gran proporción de mujeres afirmó que dependía de los ingresos de sus maridos/compañeros, de las cuales el $41 \%$ mencionó no poseer ingresos. Las entrevistadas también tenían menor dominio de idiomas $y$, aparentemente, migraban con el apoyo del marido, posiblemente con posterioridad a la venida de este. Además, la colecta de datos se realizó algunos meses después de la Copa del Mundo, cuando los contratos temporarios de trabajo habían terminado y muchos haitianos estaban nuevamente en busca de empleos o habían sido recién contratados. Entre los hombres que indicaron no poseer ingresos, muchos afirmaron estar viviendo de reservas económicas de trabajos anteriores o con la ayuda de amigos y/o familiares ${ }^{(15)}$.

De manera general, parece no haber diferencias entre sexos con relación al proyecto migratorio. Aproximadamente, el $72 \%$ de los inmigrantes residían en áreas urbanas en Haití, el 19,9\% residía en República Dominicana cuando migraron a Brasil y cerca de la mitad necesitó de ayuda financiera para migrar $(53,3 \%)$. Entre los haitianos que relataron haber recibido ayuda financiera, la mayoría la obtuvo de familiares $(67,5 \%)$ o amigos $(19,2 \%)$, pero una proporción importante recurrió a prestamistas $(13,3 \%)$. Cuando se les preguntó si poseían alguna deuda financiera relacionada con esa ayuda, el $71 \%$ respondió afirmativamente. Cabe destacar la importancia de este tipo de información para analizar el perfil del trabajador haitiano en Brasil, con deudas contraídas por el proyecto migratorio y con una gran proporción de desempleados, lo que no es posible mostrar a través de los registros oficiales disponibles, como los del Ministerio de Trabajo.

\section{Principales sectores productivos con presencia de haitianos en el estado de Mato Grosso}

El uso de la fuerza de trabajo de inmigrantes haitianos en Mato Grosso se ha dado predominantemente en eslabones de la cadena productiva del agronegocio, en la construcción civil y en el sector servicios, principales ramos responsables del desarrollo económico del estado.

Los sectores productivos con mayor absorción de haitianos (Tabla 1) son la industria de transformación y construcción civil. En la capital, el sector de construcción civil fue el que presentó el mayor número de contrataciones de haitianos en 2014 (46,0\% de los trabajadores haitianos registrados en la capital), principalmente, dentro del grupo de construcción de edificios (25,8\%). Los datos del cuestionario también indicaron que los dos principales grupos de ocupaciones ejercidas por inmigrantes haitianos en Cuiabá fueron la construcción civil y el sector de servicios. Lo que Ilama la atención es el hecho de que estos dos principales sectores, en los que están insertos los haitianos en la capital -servicios y construcción civil-, están entre las principales ramas de generación tanto de empleos como de accidentes y enfermedades ocupacionales en Brasil. En 2011, 2.884 trabajadores perdieron sus vidas durante el ejercicio de sus actividades profesionales y, en 2010, se registraron 2.753 muertes en el trabajo. El sector de servicios tuvo el mayor índice de accidentalidad en 2011, con 341.000 notificaciones de accidentes de trabajo, y en la construcción civil se produjo el mayor aumento del registro de accidentalidad con 59.808 accidentes de trabajo computados ${ }^{(33)}$.

En los municipios del interior, el sector de industrias de transformación absorbió la mayor parte de los inmigrantes $(49,7 \%)$ del mercado formal de trabajo, con el 39,8\% de los trabajadores registrados en mataderos y en la fabricación de productos de carne. La industria frigorífica forma parte de la industria de transformación y, en el caso de Mato Grosso, representa el $44,2 \%$ de toda la mano de obra empleada en el sector industrial ${ }^{(34)}$. Es 
Tabla 1. Actividad económica de los trabajadores haitianos registrados en el mercado formal de trabajo en el estado de Mato Grosso, Brasil, 2014.

\begin{tabular}{|c|c|c|c|c|c|c|}
\hline \multirow[t]{2}{*}{ Actividad económica $^{\mathrm{a}}$} & \multicolumn{2}{|c|}{$\begin{array}{c}\text { Capital }^{\mathrm{b}} \\
(\mathrm{n}=1.541)\end{array}$} & \multicolumn{2}{|c|}{$\begin{array}{c}\text { Interior } \\
(\mathrm{n}=610)\end{array}$} & \multicolumn{2}{|c|}{$\begin{array}{c}\text { Total } \\
(\mathrm{n}=2.151) \\
\end{array}$} \\
\hline & $\mathrm{n}$ & $\%$ & $\mathrm{n}$ & $\%$ & $\mathrm{n}$ & $\%$ \\
\hline Construcción & 709 & 46,0 & 158 & 25,9 & 867 & 40,3 \\
\hline Industrias de transformación & 272 & 17,7 & 303 & 49,7 & 575 & 26,7 \\
\hline Comercio; fabricación de vehículos automotores y motocicletas & 173 & 11,2 & 77 & 12,6 & 250 & 11,6 \\
\hline Alojamiento y alimentación & 93 & 6,0 & 39 & 6,4 & 132 & 6,1 \\
\hline Actividades administrativas y servicios complementarios & 111 & 7,2 & 7 & 1,1 & 118 & 5,5 \\
\hline Agua, saneamiento, actividades de gestión de residuos y descontaminación & 56 & 3,6 & - & - & 56 & 2,6 \\
\hline Transporte, almacenamiento y correo & 48 & 3,1 & 5 & 0,8 & 53 & 2,5 \\
\hline Otras actividades de servicios & 36 & 2,3 & - & - & 36 & 1,7 \\
\hline Actividades profesionales, científicas y técnicas & 16 & 1,0 & 1 & 0,2 & 17 & 0,8 \\
\hline Educación & 16 & 1,0 & - & - & 16 & 0,7 \\
\hline Agricultura, ganadería, producción forestal, pesca y acuicultura & 4 & 0,3 & 9 & 1,5 & 13 & 0,6 \\
\hline Industria extractiva & 1 & 0,1 & 3 & 0,5 & 4 & 0,2 \\
\hline Salud humana y servicios sociales & - & - & 4 & 0,7 & 4 & 0,2 \\
\hline Información y comunicación & 2 & 0,1 & 1 & 0,2 & 3 & 0,1 \\
\hline Actividades inmobiliarias & - & - & 3 & 0,5 & 3 & 0,1 \\
\hline Administración pública, defensa y seguridad social & 2 & 0,1 & - & - & 2 & 0,1 \\
\hline Artes, cultura, deporte y recreación & 2 & 0,1 & - & - & 2 & 0,1 \\
\hline
\end{tabular}

importante mencionar que el $18 \%$ de los accidentes de trabajo fatales, registrados desde 2000 a 2014 en Brasil, en trabajadores de 18 a 24 años, se concentraron en el sector agropecuario $^{(35)}$.

Este perfil de inserción de haitianos en los procesos de trabajo en Mato Grosso podría ser similar al observado a nivel nacional, cuyos principales empleadores son los sectores de industria de la transformación y construcción civil. En el año 2013, el sector de industria de transformación y de la construcción ocuparon el $3^{\circ}$ y el $4^{\circ}$ lugar en cuanto al número de trabajadores en el estado $(11,5 \%$ y $7,5 \%$, respectivamente), debajo de comercio $(29,9 \%)$ y administración pública $(19,5 \%)$. Estos datos de ocupación coinciden incluso con lo que ha sido reportado en la bibliografía internacional. Los trabajadores inmigrantes en diversas regiones del mundo están concentrados en los procesos productivos de la agricultura, la construcción civil, los servicios y la industria de la indumentaria, conocidos por el alto índice de riesgos ${ }^{(7,8)}$.

Es importante destacar que el modelo de desarrollo económico adoptado por el estado de Mato Grosso determina configuraciones sociotécnicas-ambientales de sus cadenas de producción que, a su vez, generan determinantes y condicionantes de los procesos salud-enfermedad de las poblaciones, que incluyen a los inmigrantes. El agronegocio ocupa una posición central en la economía matogrossense $y$, junto con la construcción civil, han sido responsables de conflictos socioambientales, ocupacionales y sanitarios, 
como muestran la destrucción de ecosistemas, deforestación, concentración de ingresos y tierra, aglomeración urbana, aumento de la violencia urbana y rural contra naciones indígenas y poblaciones tradicionales, superexplotación de trabajadores más pobres y de niños, altos índices de accidentes de trabajo, intoxicaciones humanas y contaminación ambiental por agrotóxicos ${ }^{(30,36,37,38)}$. Tales impactos corroen las relaciones sociales, generan gastos públicos reparatorios y constituyen daños ambientales y sanitarios que se expresan en enfermedades y muertes que podrían ser evitadas ${ }^{(39)}$.

En ese escenario, los inmigrantes haitianos están ocupando puestos de trabajos en cadenas de naturaleza "sucia", dadas sus históricas marcas en la producción de accidentes y agravios, como es el caso del sector de producción de carnes. El agronegocio ha generado una degradación social del trabajo, en el que elementos sociales, culturales y económicos conforman un entramado que crea corrientes invisibles que sujetan a los trabajadores a situaciones precarias y degradantes ${ }^{(9)}$. Los frigoríficos son una etapa importante de esa cadena del agronegocio. Este sector crece en el escenario nacional y para la contratación de trabajadores no exige escolaridad, formación, etc., apenas tener buenas condiciones de salud para desempeñar tareas repetitivas, desgastantes e insalubres, en ambientes colmados de riesgos físicos, químicos, biológicos y ergonómicos, además de un ritmo acelerado y otros aspectos de una rígida organización del trabajo $^{(40)}$. La inserción de inmigrantes haitianos puede estar cumpliendo un papel de reposición de la mano de obra para este sector que ha producido diversos problemas que causan incapacidades en la clase trabajadora ${ }^{(40)}$.

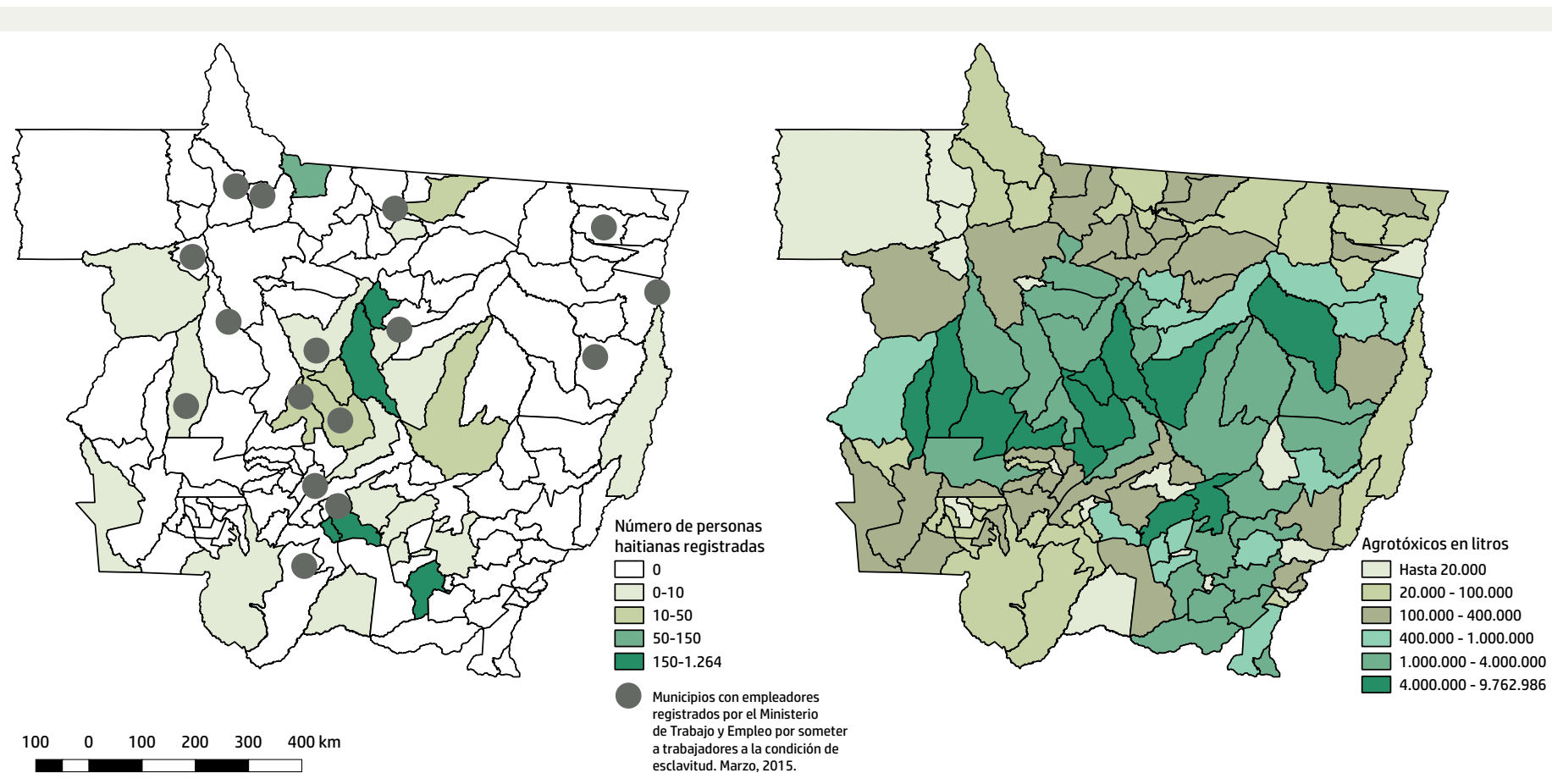

Figura 1. Número de haitianos registrados en el mercado formal de trabajo (2014), municipios con empleadores registrados por someter trabajadores a la condición de esclavitud (2015) y consumo de agrotóxico (2012), según municipios del estado de Mato Grosso, Brasil.

Fuente: Elaboración propia con base en Ministerio de Trabajo, Relación Anual de Informaciones Sociales (RAIS) ${ }^{(24)}$, Registro de empleadores que sometieron trabajadores a la condición de esclavo(27,28) e Instituto de Defensa Agropecuaria ${ }^{(29)}$. 


\section{Vulnerabilidades socioambientales de haitianos en el interior del estado y en la capital}

Más allá de la inserción en procesos productivos de alto riesgo para la salud de los trabajadores, la vulnerabilidad de la población de trabajadores inmigrantes haitianos en Mato Grosso se ha expresado: a) en su circulación por localidades con alto índice de consumo de agrotóxicos y con una historia de explotación análoga a la esclavitud y b) en quejas de actitudes discriminatorias con relación a la etnia y el color.

La condición análoga a la esclavitud se puede caracterizar por la jornada exhaustiva, trabajos forzados, condiciones degradantes o restricción del transporte. Esas formas de explotación generan agravios y enfermedades físicas y mentales como estrés postraumático, consumo excesivo de drogas y alcohol, accidentes de trabajo, entre otros ${ }^{(11)}$.

En marzo de 2015, según el Ministerio de Trabajo, en 16 municipios había empleadores registrados por someter a los trabajadores a la condición de esclavitud. En cuatro de ellos - Cuiabá, Nova Mutum, Matupá y Sapezal- había trabajadores haitianos registrados en el mercado formal (Figura 1).

En 2013, la Comisión Pastoral de la Tierra recibió denuncias contra empleadores por someter trabajadores a la condición de esclavos en ocho municipios del estado, de los cuales cinco estaban en el registro del Ministerio de Trabajo en 2015 (Matupá, Paranaíta, Ribeirão Cascalheira, Santo Antônio de Leverger e São Felix do Araguaia) y tres con registros de haitianos en la RAIS en 2014: Matupá, Paranaíta y Santo Antônio de Leverger.

Estos datos señalan que la ruta de la inmigración en Mato Grosso, además de incidir sobre ciudades del agronegocio, recorre territorios vulnerables a la esclavitud contemporánea. Este hecho es importante porque las poblaciones sometidas al trabajo esclavo son generalmente jóvenes, pobres, con baja escolaridad y migrantes ${ }^{(11)}$. La población de haitianos en Mato Grosso puede estar sujeta a esas formas laborales reductoras de las libertades, dado que la mayoría de esos inmigrantes tienen bajo nivel de escolaridad e ingresos, reflejando la realidad del país de origen. Cabe destacar aún el hecho de que algunos casos de "liberación" de haitianos del trabajo esclavo ya se produjeron en regiones de Brasil, incluso, en la capital matogrossense ${ }^{(27)}$. Cabe señalar que los territorios de atracción de empleos del interior del estado, a medida que se alejan de la capital, presentan un gradiente de situaciones de riesgo e impactos en la salud del trabajador ${ }^{(41)}$.

Sumado a esto, los resultados del cuestionario mostraron que los inmigrantes tienen deudas contraídas para llegar a Brasil. Esas deudas o el propio envío de dinero para el sustento de la familia en Haití, restringen a los trabajadores y agravan la situación de vulnerabilidad llevándolos a someterse a actividades más precarias con bajos salarios que solo alcanzan para la manutención de su propio cuerpo y el sustento de familiares en Haití. Los datos recolectados no nos permiten afirmar que se trata de casos de tráfico humano, pero no se puede descartar que existan elementos que lo constituyen, aún más considerando que las deudas contraídas colocan a estos trabajadores en situación de servidumbre frente a las relaciones y condiciones de trabajo precarias.

Otro punto importante es el alto índice de consumo de agrotóxicos en diversos municipios del interior de Mato Grosso. Muchos de estos municipios atraviesan situaciones peculiares de riesgo al estar cercados por monocultivos con múltiples exposiciones a los agrotóxicos. Llama la atención que entre los 11 municipios con mayor consumo de agrotóxicos en el proceso productivo agropecuario (superior a cuatro millones de litros), en cinco había trabajadores haitianos registrados (Sorriso, Sapezal, Nova Mutum, Nova Ubiratã y Lucas do Rio Verde). Este hecho indica que la ruta de la inmigración haitiana en Mato Grosso también atraviesa los territorios expuestos a agentes extremadamente tóxicos causantes de diversos problemas de salud de la población. Es importante resaltar que existe un aumento de los agravios relacionados con la exposición ambiental a agrotóxicos, por ejemplo, el aumento del registro de 
cánceres en las regiones de mayor consumo de agrotóxicos en el estado ${ }^{(42)}$ y de la contaminación de leche materna ${ }^{(43)}$.

A nivel internacional, los estudios también mostraron la exposición de inmigrantes a los agrotóxicos y encontraron asociación entre esa exposición y angustia y problemas psicológicos ${ }^{(10)}$. Esto implica que la población haitiana puede experimentar situaciones de vulnerabilidad socioambiental junto con las poblaciones ya expuestas a los agrotóxicos en Mato Grosso, y configurar un grupo más vulnerable, dado que los riesgos son desigualmente distribuidos y aquellos más peligrosos y perjudiciales inciden sobre las poblaciones más pobres y comunidades ne$\operatorname{gras}^{(44)}$, tal como lo muestran innumerables casos de injusticia ambiental. Generalmente, las comunidades de bajos ingresos y la población negra soportan de modo desproporcional la carga de las contaminaciones ambientales lo que muestra las inequidades sociales ${ }^{(45)}$

Esta interiorización de los inmigrantes haitianos en el estado de Mato Grosso debe ser destacada porque muchas regiones con registros de haitianos en el mercado formal son caracterizadas por la colonización sureña, sobre todo en ciudades productoras de madera, soja y algodón, donde se agudizan las desigualdades por ingresos y color. A pesar de que haya pocos trabajadores formales registrados en la agricultura, cabe sospechar que una parcela de trabajadores haitianos pueda estar prestando servicios en actividades agrícolas, contratados bajo vínculos informales por día o por tareas, que son formas de trabajo precarizado comunes en el interior del estado ${ }^{(45)}$ con un mayor grado de precarización y riesgos.

En el último censo demográfico, menos del $10 \%$ de la población de los principales municipios matogrossenses con registros de haitianos declararon ser de piel negra ${ }^{(46)}$. Estas regiones, otrora tierras de mayoría indígena, han sufrido un proceso de colonización por parte de poblaciones blancas, desde la década de 1970. La actual entrada de haitianos en estos territorios tiene implicaciones sociales y raciales y, en el transcurso de esta investigación fue posible percibir cuán expuestos están estos trabajadores inmigrantes a los discursos y actitudes xenofóbicas. La apertura de las fronteras geográficas no es simétrica a las sociales y culturales, por lo que es preciso investigar más atentamente esa relación entre los migrantes colonizadores de esas ciudades de la década de 1970 y los actuales inmigrantes de la región. Las vacantes de empleo en el mercado de trabajo para esa población negra, en sectores y funciones menos reconocidas socialmente, manifiestan elementos de prácticas discriminatorias relativas al preconcepto respecto del color de piel y al marcado racismo aún en Brasil. Esas violencias simbólicas pueden agudizar las situaciones de vulnerabilidad y riesgos para la salud de los inmigrantes, incluso porque estos factores son determinantes y contribuyen a la exclusión, el estrés y el sufrimiento de esos trabajadores ${ }^{(46)}$.

Uno de los aspectos que caracterizan a los contextos de vulnerabilidad es la "(re)producción social de grupos vulnerables, que viven en las periferias sociales, económicas y geográficas de territorios marcados por la inequidad y la discriminación, con menos recursos para protegerse de los riesgos y con bajo poder de influencia sobre procesos decisorios que definen la regulación y prevención de los riesgos" ${ }^{\prime(17)}$. Los haitianos experimentan ese aspecto dentro de un contexto vulnerable en Mato Grosso, que se suma a la vulnerabilidad social, técnica y ambiental generada por el modo de desarrollo del agronegocio. Este modelo agroexportador, fundamentado en el uso de tecnologías dañinas, como parte del desarrollo destructivo de las fuerzas productivas del capital, refuerza los mecanismos de concentración de riquezas, de tierras, de capital, de precarización laboral y de exclusión social ${ }^{(47)}$.

\section{Condiciones de trabajo, riesgos ocupacionales y manifestaciones de sufrimiento}

De modo más específico, los datos del cuestionario mostraron que, entre haitianos que 
estaban trabajando en la capital $(52,7 \%)$, una gran parte hacía entre 3 y 6 meses que estaba en el trabajo actual $(38,7 \%)$ y relataron una carga horaria semanal mayor a 48 horas $(26,5 \%)$.

Se observó que la mayoría tenía vínculo formal, aunque las mujeres presentaron un porcentaje más alto de contratos temporarios. Si bien una proporción mayor de mujeres afirmó no poseer experiencia laboral anterior $(61,9 \%)$, la mitad de los entrevistados relató haber recibido entrenamiento de seguridad laboral y el $43 \%$, algún tipo de capacitación.

La ocupación en Haití mencionada con más frecuencia fue la de trabajador en producción agrícola y solo 10 entrevistados mencionaron que estaban desempleados. Entre los hombres, el $25,2 \%$ trabajaban en la agricultura, el $17,4 \%$ eran estudiantes y el oficio de albañil fue el tercero más citado $(12,1 \%)$. Entre las mujeres, la mayoría mencionó ser comerciante $(60,8 \%)$. Ya en Brasil, la mayoría de los hombres que tenía trabajo ocupaba puestos de ayudante de albañil $(48,7 \%)$ y más de la mitad de las mujeres $(53,5 \%)$ conformaba el grupo de personal de limpieza (Tabla 2).

Existen claros desencuentros y discrepancias entre las profesiones y tareas realizadas en Haití y en Brasil. Se observó que, generalmente, la formación, la experiencia y la profesión ejercida en Haití eran muy diferentes a la ocupación en Mato Grosso. Además, hay haitianos calificados que ejercen funciones muy por debajo de sus capacidades, debido a la falta de revalidación de diplomas.

Estos hechos, aparentemente sin mayor importancia, pueden revelar situaciones

Tabla 2. Principales ocupaciones en Haití y en Brasil, ejercidas por los inmigrantes haitianos residentes en Cuiabá, Mato Grosso, Brasil, 2015.

\begin{tabular}{|c|c|c|c|c|c|}
\hline \multicolumn{6}{|c|}{ Principales ocupaciones ejercidas en Haití } \\
\hline \multicolumn{3}{|c|}{ Hombres $(n=373)$} & \multicolumn{3}{|c|}{ Mujeres $(n=79)$} \\
\hline Ocupaciones & $\mathrm{n}$ & $\%$ & Ocupaciones & $\mathrm{n}$ & $\%$ \\
\hline Trabajador agrícola temporal & 94 & 25,2 & Comerciante minorista & 48 & 60,8 \\
\hline Estudiante & 65 & 17,4 & Estudiante & 7 & 8,9 \\
\hline Albañil & 45 & 12,1 & Esteticista & 5 & 6,3 \\
\hline Comerciante minorista & 23 & 6,2 & Costurera & 5 & 6,3 \\
\hline Ayudante de albañil & 22 & 5,9 & Profesora & 3 & 3,8 \\
\hline Conductor (taxista) & 22 & 5,9 & Personal de limpieza & 2 & 2,5 \\
\hline Profesor & 12 & 3,2 & Trabajadora agrícola temporal & 2 & 2,5 \\
\hline Desempleado & 9 & 2,4 & Desempleada & 1 & 1,3 \\
\hline Otras & 81 & 21,7 & Otras & 6 & 7,5 \\
\hline \multicolumn{6}{|c|}{ Principales ocupaciones ejercidas en Brasil (actual) } \\
\hline \multicolumn{3}{|c|}{ Hombres (n=195) } & \multicolumn{3}{|c|}{ Mujeres $(n=43)$} \\
\hline Ocupaciones & $\mathrm{n}$ & $\%$ & Ocupaciones & $\mathrm{n}$ & $\%$ \\
\hline Empleado de obra & 95 & 48,7 & Personal de limpieza & 23 & 53,5 \\
\hline Personal de limpieza & 31 & 15,9 & Cocinera general & 5 & 11,6 \\
\hline Albañil & 27 & 13,8 & Empleada de obras & 5 & 11,6 \\
\hline Plomero & 9 & 4,6 & Lavandera en general & 4 & 9,3 \\
\hline Costurero & 3 & 1,5 & Repositora de mercadería & 2 & 4,7 \\
\hline Lavandero en general & 2 & 1,0 & Otras & 4 & 9,3 \\
\hline Repositor de mercadería & 2 & 1,0 & & & \\
\hline Otras & 26 & 13,3 & & & \\
\hline
\end{tabular}


complejas porque son cuestiones que afectan la identidad y la salud mental de los inmigrantes. Según la psicodinámica del trabajo, por ejemplo, las crisis de salud mental de los trabajadores son también crisis de identidad, eso porque el trabajo ocupa un lugar fundamental en la economía simbólica, en la salud mental y en la propia identidad de los trabajadores $^{(21)}$. Por eso, el trabajo proporciona placer y también es fuente de sufrimientos intensos. Es importante mencionar que, de acuerdo con un relevamiento realizado por Porru, Elmetti y Arici ${ }^{(48)}$, aún es bajo el número de publicaciones sobre salud mental de trabajadores inmigrantes, por lo que es necesario considerar que la identidad construida en el lugar (en este caso, en Haití) y toda la gama de experiencias y saberes movilizados en los trabajadores a partir de sus ocupaciones entran en choque, aunque de manera momentánea, en el proceso de desterritorialización y reterritorialización ${ }^{(49)}$, donde se asumen nuevas actividades.

\section{Desterritorialización es, frecuentemente, otra palabra para expresar extraña- miento, que es, también, desculturiza- ción. [...] Cuando el hombre se enfrenta a un espacio que no ayudó a crear, cuya historia desconoce, cuya memoria le es extraña, ese lugar es la sede de una fuerte alienación. ${ }^{(49)}$}

El ser humano es también una formación de elementos configurados en los territorios, por eso, "la 'residencia', el lugar de trabajo, por más breve que sean, son marcos de vida que tienen peso en la producción del hombre"(49).

En los dos grandes grupos de ocupaciones ejercidas por estos inmigrantes en Cuiabá (construcción civil y el sector de servicios), los principales riesgos percibidos fueron físicos $(53,2 \%$ y $63,4 \%$, respectivamente) y de accidentes $(23,4 \%$ y $17,1 \%$, respectivamente). Se observó también el relato de agrupamientos de riesgos, o sea, trabajadores que relataron percibir más de un riesgo (físicos y químicos o físicos y de accidentes). Menos de la mitad de los entrevistados relató capacitación previa para la tarea que realizaba. Además, el
$62,5 \%$ de los trabajadores de la construcción civil y el $55,2 \%$ del sector de servicios mencionaron haber participado de algún tipo de formación en seguridad laboral.

A pesar de no haber diferencias entre la proporción de hombres y mujeres que trabajaban en Cuiabá al momento de la investigación, las mujeres recibían una menor remuneración salarial, menos entrenamiento en seguridad laboral y no tenían experiencia en las actividades que ejercían. Estos datos refuerzan la aparente mayor vulnerabilidad de las mujeres que puede estar vinculada a la división sexual del trabajo.

Respecto a lo que más les incomodaba en la ejecución de las actividades, entre los haitianos entrevistados que estaban trabajando, 90 mencionaron quejas referentes a las dificultades de lidiar con los patrones y gestores, no trabajar en la función adecuada y a la gestión y organización del trabajo, destacando la falta de reconocimiento y valorización por parte de la empresa, a la que "no les gustan los extranjeros". El elemento que más sobresalió fue la remuneración, pues 24 de ellos manifestaron insatisfacción en cuanto a los salarios que son "muy bajos", además de atrasos y hasta falta de pago.

Otro grupo de quejas se centró en el sufrimiento físico y psicosocial: dolores en la columna, cabeza, estómago, o en todo el cuerpo, además de esfuerzo intenso, cansancio, inseguridad en la ejecución de actividades, etc. También mencionaron intimidación, humillación, falta de respeto y nostalgia de la familia, conforme la expresión de un trabajador inmigrante: "en todo momento pienso en mi familia". En ese sentido, ciertas investigaciones señalan problemas de salud mental en trabajadores migrantes, como casos de depresión ocasionados por la "soledad asociada a la larga y exhaustiva jornada de trabajo", silenciamiento sobre los padecimientos y el miedo de perder el empleo y no conseguir mantenerse lejos de casa ${ }^{(50)}$.

Resulta llamativa la extensa jornada de trabajo a la que estaban sometidos. A pesar de que los trabajadores no hayan mencionado en sus relatos la extensa carga horaria como algo que los incomodaba, se percibieron 
los efectos de ocupaciones con elevada exigencia física, realizadas por largos períodos, con el relato de manifestaciones (dolores en el cuerpo y cansancio). Estos elementos interactúan con el cuerpo de los trabajadores generando pérdidas de las capacidades potenciales corporales y psíquicas ${ }^{(14)}$.

Otro punto importante es el número de inmigrantes desempleados en la capital matogrossense $(47,3 \%$ de los haitianos entrevistados). Dada la centralidad del trabajo en los fenómenos migratorios y en la salud mental de los trabajadores, tal como lo señala Dejours y Molinier ${ }^{(21)}$, y considerando además que muchos haitianos fueron a Brasil con la expectativa de conseguir trabajo ${ }^{(51)}$, esa situación puede llevar a la percepción de un deterioro de la salud y a experimentar disturbios mentales como lo muestran estudios realizados en diversos contextos mundiales ${ }^{(7,8)}$.

\section{CONSIDERACIONES FINALES}

Este artículo abordó la inserción de trabajadores inmigrantes en procesos de trabajo en Mato Grosso, considerando sus condiciones laborales, situaciones de riesgo y vulnerabilidades socioambientales a la luz del contexto económico del estado y de los aspectos socioeconómicos de este grupo poblacional. Los resultados indican la fragilidad de la inserción social de esa población. Esa fragilidad se expresa tanto en la situación del desempleo como en la presencia de haitianos en áreas y procesos productivos de alto riesgo socioambiental y que impactan en la salud de esa población. Brasil supone un ambiente social y político atractivo para la migración internacional de trabajadores de países pobres para ocupar puestos de trabajos en áreas vulnerables y en cadenas productivas con alto riesgo para la salud del trabajador. Sobre la base de lo detectado en este trabajo, estudios futuros deberían caracterizar las condiciones de trabajo de haitianos del interior del estado e identificar situaciones de riesgos, agravios y daños a la salud de trabajadores inmigrantes delimitados por rama productiva.
La compleja y dinámica articulación entre migración, procesos de trabajo, situaciones de riesgo y vulnerabilidades socioambientales puede ser comprendida como expresión del actual escenario económico y modelo de desarrollo adoptado en Brasil, marcado por la exportación de commodities agrícolas reflejado en Mato Grosso. Así, al caracterizar un nuevo grupo de trabajadores vulnerables en el contexto de las relaciones salud-trabajo, este estudio advierte sobre el desafío de la salud pública y demás sectores del Estado en la universalización de derechos, sobre todo en ese momento histórico de crisis mundial en la cuestión migratoria. Esto requiere más acciones institucionales públicas, nacionales y locales para la defensa de la salud, dignidad y mejora de las condiciones de trabajo de esa población en Brasil, por ejemplo, de los sectores de Previsión Social, Trabajo y Empleo, Ministerio Público, entre otros, y de la sociedad civil y los movimientos sociales.

Cabe señalar la responsabilidad e importancia de la vigilancia de la salud del trabajador en la identificación de las características específicas de este grupo poblacional y las peculiaridades del proceso de adaptación a la nueva lengua, costumbres, cultura y de su inserción en procesos productivos generadores de riesgos y vulnerabilidades, para articular acciones intersectoriales y participativas en pro de la salud. Esto requiere que esta temática sea incluida en los programas y acciones de los centros de referencia en salud del trabajador, para generar estrategias y formas de realizar vigilancia conectadas a la atención integral de la salud de los inmigrantes para la implementación efectiva de la Política Nacional de Salud del Trabajador y de la Trabajadora que prioriza a grupos de trabajadores más vulnerables.

Debemos destacar que la generalización de la tercerización y el recrudecimiento del trabajo esclavo en Brasil en 2017, tiende a agravar la precarización del trabajo y los impactos en la salud, aumentando así las situaciones que generan condiciones de desaliento y de fuerte daño social a grupos vulnerables, como los inmigrantes. 


\section{REFERENCIAS BIBLIOGRÁFICAS}

1. Dutra D, Almeida S, Tonhati T, Palermo G. Os estrangeiros no mercado de trabalho formal brasileiro: perfil geral na série 2011, 2012 e 2013. Cadernos OBMigra-Revista Migrações Internacionais. 2015;1(2):2359-5337.

2. UNHCR, ACNUR. Refúgio no Brasil: uma análise estatística; janeiro de 2010 a outubro de 2014 [Internet]. Brasil: Alto Comisionado de las Naciones Unidas para los Refugiados; 2014 [citado 18 ene 2016]. Disponible en: https://tinyurl.com/ yamlnsqh.

3. Cavalcanti L. Imigração e mercado de trabalho no Brasil: características e tendências. Cadernos OBMigra-Revista Migrações Internacionais. 2015;1 (2):35-47.

4. Walia $\mathrm{H}$. Transient servitude: migrant labour in Canada and the apartheid of citizenship. Race \& Class. 2010;52(1):71-84.

5. Sennett R. A cultura do novo capitalismo. Rio de Janeiro: Record; 2006.

6. Siqueira CE, Gaydos M, Monforton C, Slatin C, Borkowski L, Dooley P, Liebman A, Rosenberg E, Shor G, Keifer M. Effects of social, economic, and labor policies on occupational health disparities. American Journal of Industrial Medicine. 2014;57(5):557-572.

7. Benach J, Muntaner C, Chung H, Benavides FG. Immigration, employment relations, and health: developing a research agenda. American Journal of Industrial Medicine. 2010;53(4):338-343.

8. Ahonen EQ, Benavides FG, Benach J. Immigrant populations, work and health: a systematic literature review. Scandinavian Journal of Work, Environment \& Health. 2007;33(2):96-104.

9. Silva MAM, Martins RC. A degradação social do trabalho e da natureza no contexto da monocultura canavieira paulista. Sociedade e Estado. 2010;12(24):196-240.

10. Griffin J, Soskolne V. Psychological distress among Thai migrant workers in Israel. Social Science \& Medicine. 2003;57(5):769-774.

11. Leão LHC. Trabalho escravo contemporâneo como um problema de saúde pública. Ciência \& Saúde Coletiva. 2016;21(12):3927-3936.

12. Make the Road New York. Immigrant worker health and safety: a guide for front-line advocates [Internet]. New York: Occupational Safety and Health Administration, US Department of Labor;
2011 [citado 18 ene 2016]. Disponible en: https:// tinyurl.com/y8hfru8a.

13. Minayo-Gomez C, Thedim-Costa SMF. A construção do campo da saúde do trabalhador: percurso e dilemas. Cadernos de Saúde Pública. 1997;13(Suppl 2):21-32.

14. Laurell AC, Noriega M. Processo de produção e saúde: trabalho e desgaste operário. São Paulo: Hucitec; 1989.

15. Leão LHC, Muraro AP, Palos CC, Martins MAC, Borges FT. Migração internacional, saúde e trabalho: uma análise sobre os haitianos em Mato Grosso, Brasil. Cadernos de Saúde Pública. 2017;33(7):e00181816.

16. Borges FT, Muraro AP, Leão LHC, Carvalho LA, Siqueira CEG. Socioeconomic and health profile of Haitian immigrants in a Brazilian Amazon State. Journal of Immigrant and Minority Health. 2018. doi: 10.1007/s10903-018-0694-9.

17. Batista DRR, Gugelmin SA, Muraro AP. Prenatal follow-up of Haitian and Brazilian women in Mato Grosso. Revista Brasileira de Saúde Materno Infantil. 2018;18(2):317-326.

18. Porto MFS. Uma ecologia política dos riscos: princípios para integrarmos o local e o global na promoção da saúde e da justiça ambiental. Rio de Janeiro: FIOCRUZ; 2012.

19. Cartier R, Barcellos C, Hübner C, Porto MF. Vulnerabilidade social e risco ambiental: uma abordagem metodológica para avaliação de injustiça ambiental. Cadernos de Saúde Pública. 2009;25(12):2695-2704.

20. Bourdieu P, Passeron JC. A reprodução: elementos para uma teoria do sistema de ensino. Rio de Janeiro: Fracisco Alves Editora; 1992.

21. Dejours C, Molinier P. O trabalho como enigma. En: Lancman S, Sznelwar L, (org.). Christophe Dejours: da psicopatologia à psicodinâmica do trabalho. Brasília: Paralelo 15 Editores; 2004.

22. Krieger N. Discrimination and health. En: Berkman LF, Kawachi I. Social Epidemiology. New York: Oxford University Press; 2000. p. 36-75.

23. Lwanga SK, Lemeshow S. Sample size determination in health studies: a practical manual. Geneva: World Health Organization; 1991.

24. Brasil, Ministério do Trabalho. Relação Anual de Informações Sociais [Internet]. c2018 [citado 18 ene 2016]. Disponible en: https://tinyurl.com/ ybbu94qx. 
25. Brasil; Ministério do Planejamento, Orçamento e Gestão. Resolução CONCLA No 2/2010 [Internet]. 8 jun 2010 [citado 18 ene 2016]. Disponible en: https://tinyurl.com/y7r9jeey.

26. Brasil, Ministério do Trabalho. Classificação Brasileira de Ocupações [Internet]. 2002 [citado 18 ene 2016]. Disponible en: https://bit.ly/1pO9ADe.

27. Comissão Pastoral da Terra, Secretaria Nacional. Campanha da CPT de Combate ao Trabalho Escravo divulga dados de 2013 [Internet]. Goiás: Comissão Pastoral da Terra, Secretaria Nacional; 2013 [citado 10 nov 2015]. Disponible en: https://tinyurl.com/yc4toaaq.

28. Wrobleski S. Imigrantes haitianos são escravizados no Brasil [Internet]. Reporter Brasil; 2014 [citado 10 nov 2015]. Disponible en: https:// tinyurl.com/yaj7xyug.

29. Instituto de Defesa Agropecuária do Mato Grosso. Relatório-planilha de dados do sistema de informação de agrotóxicos dos anos de 2005 a 2012. Cuiabá: Instituto de Defesa Agropecuária do Mato Grosso; 2013.

30. Pignati W, Oliveira NP, Silva AMC. Vigilância aos agrotóxicos: quantificação do uso e previsão de impactos na saúde-trabalho-ambiente para os municípios brasileiros. Ciência \& Saúde Coletiva. 2014;19(12):4669-4678.

31. Véran J-F, Noal DS, Fainstat T. Nem refugiados, nem migrantes: a chegada dos haitianos à cidade de Tabatinga (Amazonas). Dados. 2014;57(4):10071041.

32. Santos FV. A inclusão dos migrantes internacionais nas políticas do sistema de saúde brasileiro: o caso dos haitianos no Amazonas. História, Ciências, Saúde-Manguinhos. 2016;23(2):477-494.

33. Revista Proteção. Estatísticas de acidentes Brasil. En: Anuário Brasileiro de Proteção 2013 [Internet]. Novo Hamburgo: Revista Proteção; 2013 [citado 10 nov 2015]. Disponible en: https://tinyurl. com/y8sjcmv3.

34. Instituto de Geografia e Estatística. Cadastro central de empresas de 2013 [Internet]. Sistema IBGE de Recuperação Automática; 2015 [citado 10 nov 2015]. Disponible en: https://tinyurl.com/ybskrjy9.

35. Programa Integrado em Saúde Ambiental e do Trabalhador. Boletim epidemiológico acidentes de trabalho fatais em crianças e jovens de 10 a 24 anos no Brasil, 2000-2014 [Internet]. No. 10. Salvador Bahia: MS, SVS, DSAST, CGST; 2017 [citado 10 ene 2017]. Disponible en: https:// tinyurl.com/yaqqd9n8.
36. Silva MJ, Sato MT. Territórios em tensão: o mapeamento dos conflitos socioambientais do estado de Mato Grosso-Brasil. Ambiente \& Sociedade. 2012;15(1):1-22.

37. Pignati WA, Machado JMH. O agronegócio e seus impactos na saúde dos trabalhadores e da população do Estado de Mato Grosso. En: Gomez CM, Machado JHM, Pena PG, (orgs.). Saúde do trabalhador na sociedade brasileira contemporânea Rio de Janeiro: Fiocruz; 2011. p. 245-272.

38. Vasconcellos MC, Pignatti MG, Pignati WA. Emprego e acidentes de trabalho na indústria frigorífica em áreas de expansão do agronegócio, Mato Grosso, Brasil. Saúde e Sociedade. 2009;18(4):662-672.

39. Porto MF, Soares WL. Modelo de desenvolvimento, agrotóxicos e saúde: um panorama da realidade agrícola brasileira e propostas para uma agenda de pesquisa inovadora. Revista Brasileira de Saúde Ocupacional. 2012;37(125):17-31.

40. Oliveira PAB, Mendes JMR. Processo de trabalho e condições de trabalho em frigoríficos de aves: relato de uma experiência de vigilância em saúde do trabalhador. Ciência \& Saúde Coletiva. 2014;19(12):4627-4635.

41. Pignati WA, Machado JMH. Riscos e agravos à saúde e à vida dos trabalhadores das indústrias madeireiras de Mato Grosso. Ciência \& Saúde Coletiva. 2005;10(4):961-973.

42. Cunha MLON. Mortalidade por câncer e a utilização de pesticidas no estado de Mato Grosso no Período de 1998 a 2006. [Dissertação de Mestrado]. São Paulo: Faculdade de Ciências Médicas, Santa Casa de São Paulo; 2010.

43. Palma DCA, Lourencetti C, Uecker ME, Mello PRB, Pignati WA, Dores EFGC. Simultaneous determination of different classes of pesticides in breast milk by solid-phase dispersion and GC/ ECD. Journal of the Brazilian Chemical Society. 2014;25(8):1419-1430.

44. Bullard RD. Unequal protection: environmental justices and communities of color. San Francisco: Sierra Club Books; 1996.

45. Instituto Brasileiro de Geografia e Estatística Censo 2010. Instituto Brasileiro de Geografia e Estatística [Internet]; 2010 [citado 2 nov 2015]. Disponible en: https://censo2010.ibge.gov.br/.

46. Ramos N. Saúde, migração e direitos humanos. Mudanças-Psicologia da Saúde. 2009;17(1):1-11.

47. Thomaz Junior A. Dinâmica geográfica do trabalho no século XXI: limites explicativos, auto- 
crítica e desafios teóricos. [Tese de livre-docência]. São Paulo: Universidade Estadual Paulista Júlio de Mesquita Filho, Faculdade de Ciências e Tecnologia; 2009.

48. Porru S, Elmetti S, Arici C. Psychosocial risk among migrant workers: what we can learn from literature and field experiences. La Medicina del Lavoro. 2014;105(2):109-129.

49. Santos M. A natureza do espaço: técnica e tempo, razão e emoção. São Paulo: Editora da Universidade de São Paulo; 2006.
50. Menezes MA, Silva MS. A cana judia de nós: impactos da migração e da atividade de cortar cana sobre a saúde dos trabalhadores migrantes nordestinos. Em: VIII Congresso do ALASRU; 2010; Porto de Galinhas.

51. Cotinguiba GC. Imigração haitiana para o Brasil-a relação entre trabalho e processos migratórios. [Dissertação de Mestrado]. Porto Velho: Universidade Federal de Rondônia; 2014. 